\title{
Multistep activation of the Helicobacter pylori effector CagA
}

\author{
Anne Müller \\ Institute of Molecular Cancer Research, University of Zürich, Zürich, Switzerland.
}

\begin{abstract}
Chronic infection with the Gram-negative bacterium Helicobacter pylori is a major risk factor for the development of gastric cancer. Accumulating evidence indicates that the $H$. pylori virulence determinant cytotoxin-associated gene $\mathrm{A}(\mathrm{CagA})$ has a key oncogenic role in the process. Certain biological activities of CagA require its tyrosine phosphorylation by host cell kinases. In this issue of the JCI, Mueller and colleagues report their detailed kinetic and functional analysis of CagA phosphorylation, which indicates that c-Src and $\mathrm{c}-\mathrm{Abl}$ kinases sequentially phosphorylate CagA. Interestingly, the two phosphorylation events need not occur on the same CagA molecule but are both required for the biological effects of CagA. The results provide a clinically relevant example of how a successful bacterial pathogen has evolved to exploit the tightly coordinated, sequential activity of host cell kinases for virulence factor activation and induction of pathology.
\end{abstract}

\section{The role of $\boldsymbol{H}$. pylori and its virulence determinant CagA in gastric carcinogenesis}

Gastric carcinoma is the second most common cause of cancer-related mortality worldwide (1). Chronic infection with the bacterial pathogen Helicobacter pylori is a key risk factor for the development of gastric cancer (2). Individuals infected with $H$. pylori strains harboring the virulence determinant cytotoxin-associated gene A (CagA) are exposed to a substantially greater risk of developing gastric cancer than carriers of CagA-negative strains (3, 4). Experimental infection of Mongolian gerbils (5) and C57BL/6 mice (6) with isogenic strains of $H$. pylori differing only in their ability to deliver CagA to host cells confirmed the contribution of this virulence factor to severe disease outcomes. Moreover, transgenic expression of CagA in mice results in the development of gastrointestinal and hematological malignancies (7), providing further support for the notion that CagA is a bacterial oncoprotein.

CagA is encoded in the H. pylori genome as part of a $40-\mathrm{kb}$ stretch of DNA known as the cag pathogenicity island (PAI), which encodes a type IV secretion system (8). CagA is the only known protein substrate of the secretion system, which is believed to form

Conflict of interest: The author has declared that no conflict of interest exists.

Citation for this article: J Clin Invest. 2012; 122(4):1192-1195. doi:10.1172/JCI61578. a syringe-like structure protruding from the bacterial surface that directly delivers CagA to the host cell cytosol upon pathogen/ cell contact (9). Inside the eukaryotic cell, CagA is tyrosine phosphorylated on C-terminal Glu-Pro-Ile-Tyr-Ala (EPIYA) motifs by Src and Abl family kinases $(10,11)$. EPIYA motifs are found in highly polymorphic regions of CagA termed EPIYA segments A-D (Figure 1) (12). cag PAI-positive $H$. pylori strains isolated from individuals living in the Western hemisphere typically contain a tandem arrangement of A, B, and $\mathrm{C}$ segments in the $\mathrm{C}$ terminus of CagA, which usually consists of one copy each of $\mathrm{A}$ and $\mathrm{B}$, and one to three - or more - copies of $\mathrm{C}$. The generally more virulent East Asian $H$. pylori isolates possess a unique EPIYA-containing sequence, the $\mathrm{D}$ segment, which is present in one or multiple copies and arranged in tandem with $\mathrm{A}$ and B segments.

Upon tyrosine phosphorylation in the host cell, CagA binds to and aberrantly activates SHP-2 (13), a protein tyrosine phosphatase normally involved in activation of the Ras/Erk signaling pathway upon growth factor binding to its receptor. Active SHP-2 may induce cytoskeletal rearrangements via dephosphorylation of focal adhesion kinase (FAK), which results in morphological changes such as cellular elongation and increased cell motility (13). In addition to its phosphorylationdependent interaction with SHP-2 and other signaling molecules such as Csk (14) and PI3K (15), CagA is known to disrupt tight junctions between neighboring cells in a polarized epithelial monolayer and to thereby cause the loss of epithelial polarity (16). This CagA activity is phosphorylation independent and mediated by its interaction with, and inhibition of, the master regulator of cell polarity, PAR1b (17). The combined phosphorylation-dependent and -independent effects of CagA have been postulated to destroy the normal gastric mucosal architecture and to impair its barrier function, and may thus contribute to the progressive histopathological changes preceding gastric cancer.

\section{Src and Abl kinases sequentially phosphorylate CagA at two EPIYA motifs}

Substantial progress has been made in the past $12-15$ years in elucidating the biological consequences of CagA translocation and phosphorylation in the context of gastric inflammation and carcinogenesis. However, surprisingly little is known about the sequence and regulation of CagA phosphorylation events and the relative contribution of the two kinases involved. In this issue of the JCI, Mueller and colleagues have now comprehensively addressed these issues using site-directed mutagenesis, inhibitor studies, and twodimensional (2D) protein electrophoresis approaches (18).

In their first set of experiments, Mueller et al. investigated the kinetics and order of CagA phosphorylation events (18). Confirming previous results (11), they found that c-Src was activated transiently and early in infection. Strong c-Abl activation, in contrast, was only detected later after infection. 2D phosphotyrosine-specific Western blotting revealed the consecutive appearance of two distinct species of phosphorylated CagA $\left(\mathrm{CagA}^{\mathrm{PY}}\right)$, which were differentially sensitive to c-Src and c-Abl inhibition. The $\mathrm{CagA}^{\mathrm{PY}}$ spot appearing early after infection was attenuated under conditions of c-Src inhibition, but not c-Abl inhibition, whereas the two CagA $\mathrm{A}^{\mathrm{PY}}$ 


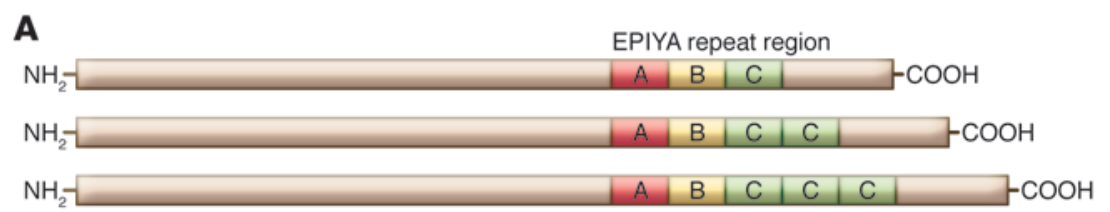

B

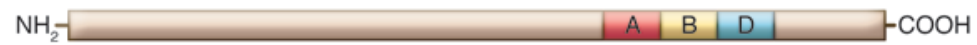

Figure 1

Schematic representation of the most common Western and East Asian CagA variants. A, B, $C$, and $D$ segments are characterized by the upstream and downstream nucleotide sequences flanking the EPIYA motif. (A) Three commonly occurring Western CagA variants. (B) A common East Asian CagA variant. Additional EPIYA configurations found in Western CagA proteins include $A B, A C, A C C, A C C C, A B C C C C, A B C C C C C, A B C A B C, A B C B C C, A B A B C, A B B B C$, $A B B C C, A A B C, A B B C, B C, C C$, and $C$. Other East Asian CagA proteins exhibit the configurations $A A B D, A B D D, A B B B D, A B D A B D, A B A B D, A B D B D, A B B D, B A B D, A D$, and $B D$.

signals characteristic of late infection were prevented by c-Abl inhibition. The results are thus consistent with an early c-Src-mediated phosphorylation event at a single EPIYA motif followed by multiple c-Abl-mediated events at two motifs.

The introduction of nonphosphorylatable $\mathrm{Y}>\mathrm{F}$ point mutations in one, two, or all three EPIYA motifs of a Western $H$. pylori strain revealed that c-Src can only phosphorylate CagA at EPIYA motifs in $\mathrm{C}$ segments, whereas c-Abl has the ability to phosphorylate EPIYA motifs in A, $\mathrm{B}$, and $\mathrm{C}$ segments. Complementation experiments in which single, double, and triple EPIYA $^{\mathrm{Y}>\mathrm{F}}$ mutant CagA proteins were expressed in CagA-deficient H. pylori also confirmed a central role for EPIYA-C phosphorylation in mediating the downstream effects of CagA $\mathrm{A}^{\mathrm{PY}}$. The cell elongation phenotype that is characteristic of CagA phosphorylation failed to develop in cells with mutant EPIYA- $\mathrm{C}^{\mathrm{Y}>\mathrm{F}}$; on the other hand, exclusive phosphorylation of EPIYA-C (in EPIYA-AB ${ }^{\mathrm{Y}>\mathrm{F}}$ mutants) was not sufficient to induce the cell elongation phenotype. Together, these data suggest that none of the three motifs are alone sufficient to activate the signaling pathways downstream of phosphorylated CagA and that consecutive phosphorylation of EPIYA-C by c-Src followed by phosphorylation of EPIYA-A or EPIYA-B by c-Abl is needed (Figure 2). The analysis of phosphorylation events in a prototypical East Asian ABD strain revealed that EPIYA-D is functionally equivalent to EPIYA-C in Western strains. The results are reminiscent of multisite, processive phosphorylation of eukaryotic proteins such as glycogen synthase kinase 3 (GSK3) and VASP by cooperating kinases, in the course of which phosphorylation at one residue by one kinase primes subsequent phosphorylation events by a second kinase at one or several nearby sites (19).

\section{CagA phosphomimetics elucidate the phosphorylation requirements for signaling downstream of CagA $\mathrm{AY}^{\mathrm{PY}}$}

To address the question of which of the three possible phosphorylation events in a typical Western ABC-type CagA triggers the downstream effects associated with CagA delivery and phosphorylation, Mueller et al. chose the laborious but informative approach of constructing a set of nine different phosphomimetic CagA mutants (18) in which the effects of tyrosine phosphorylation events are mimicked by the negatively charged side chains of aspartic acid residues. The authors replaced the tyrosines in one, two, or all three EPIYA motifs by aspartic acid residues, mutated all other EPIYA tyrosines to phenylalanines to prevent their phosphorylation, and expressed the constructs in CagAdeficient $H$. pylori. In line with previous reports of $\mathrm{Cag}^{\mathrm{PY}}$ interactors (13-15), the phosphomimetic of EPIYA-A (EPIYA-A ${ }^{\mathrm{Y}>\mathrm{D}}$ ) bound to Csk, whereas EPIYA-B ${ }^{\mathrm{P}>\mathrm{D}}$ and EPIYA-C $\mathrm{C}^{\mathrm{D} D \mathrm{D}}$ bound to PI3K and SHP-2, respectively (Figure 2). The cell elongation phenotype was induced at close to wild-type levels by bacteria expressing EPIYA-AC $\mathrm{C}^{\mathrm{Y}>\mathrm{D}}$ and EPIYA-BC ${ }^{\mathrm{Y}>\mathrm{D}}$ double phosphomimetics, but not by single mimetics or a triple mimetic. The results are thus in line with the requirement for two consecutive phosphorylation events at EPIYA motifs $C$ and $\mathrm{B}$, or $\mathrm{C}$ and $\mathrm{A}$, and explain why triplephosphorylated CagA protein species are not found in infected host cells.
The examination of numerous Western and East Asian patient isolates expressing CagA proteins with various common EPIYA configurations revealed that EPIYA-AC and EPIYA-BD CagA are phosphorylated at similar levels (and induce comparable downstream effects) as EPIYA-ABC/EPIYA-ABD CagA, whereas EPIYA-AB, - $B C$, and - $A D$ configurations are inactive in this respect. In a final set of experiments, Mueller et al. coinfected epithelial cells with two mutant $H$. pylori strains, each of which expressed just one phosphorylatable or phosphomimetic CagA motif. The bottom line of these experiments was that only the $\mathrm{A}+\mathrm{C}$ combination induced cellular elongation as efficiently as the wild-type $\mathrm{ABC}$ configuration; the $\mathrm{B}+\mathrm{C}$ combination had a severe defect, and the $\mathrm{A}+\mathrm{B}$ combination had a minor defect in inducing cellular elongation relative to the wild-type configuration.

\section{Conclusions and perspectives}

The main findings of Mueller and colleagues (18) can thus be summarized as follows (Figure 2). The two kinases c-Src and c-Abl phosphorylate CagA sequentially, and in a tightly regulated and coordinated manner. c-Src is the first kinase to become activated upon cytoplasmic CagA delivery by the cag PAI-encoded type IV secretion system. It phosphorylates CagA exclusively on EPIYA motifs flanked by C/D segment sequences; this first phosphorylation event primes the subsequent c-Abl-mediated phosphorylation of EPIYA-A, -B, -C, or -D motifs later in infection. One molecule of CagA is never phosphorylated on more than two EPIYA motifs simultaneously, and phosphorylation of a single motif is not sufficient to induce downstream effects. Interestingly, the preferred combination of phosphorylatable motifs is $\mathrm{A}+\mathrm{C}$ (and B+D in East Asian strains), which, strikingly, may be located on separate CagA molecules. The study by Mueller et al. (18) thus provides numerous novel insights into how the activity of secreted bacterial effector molecules - of which CagA remains the best understood - may be regulated by eukaryotic host cell kinases, and adds new evidence to the notion of processive phosphorylation as a key regulatory mechanism of eukaryotic signaling pathways. Whether the concepts formulated for CagA by Mueller et al. (18) hold true for other effectors secreted by bacterial type IV secretion systems, such as the BepD, -E, and -F proteins of Bar- 


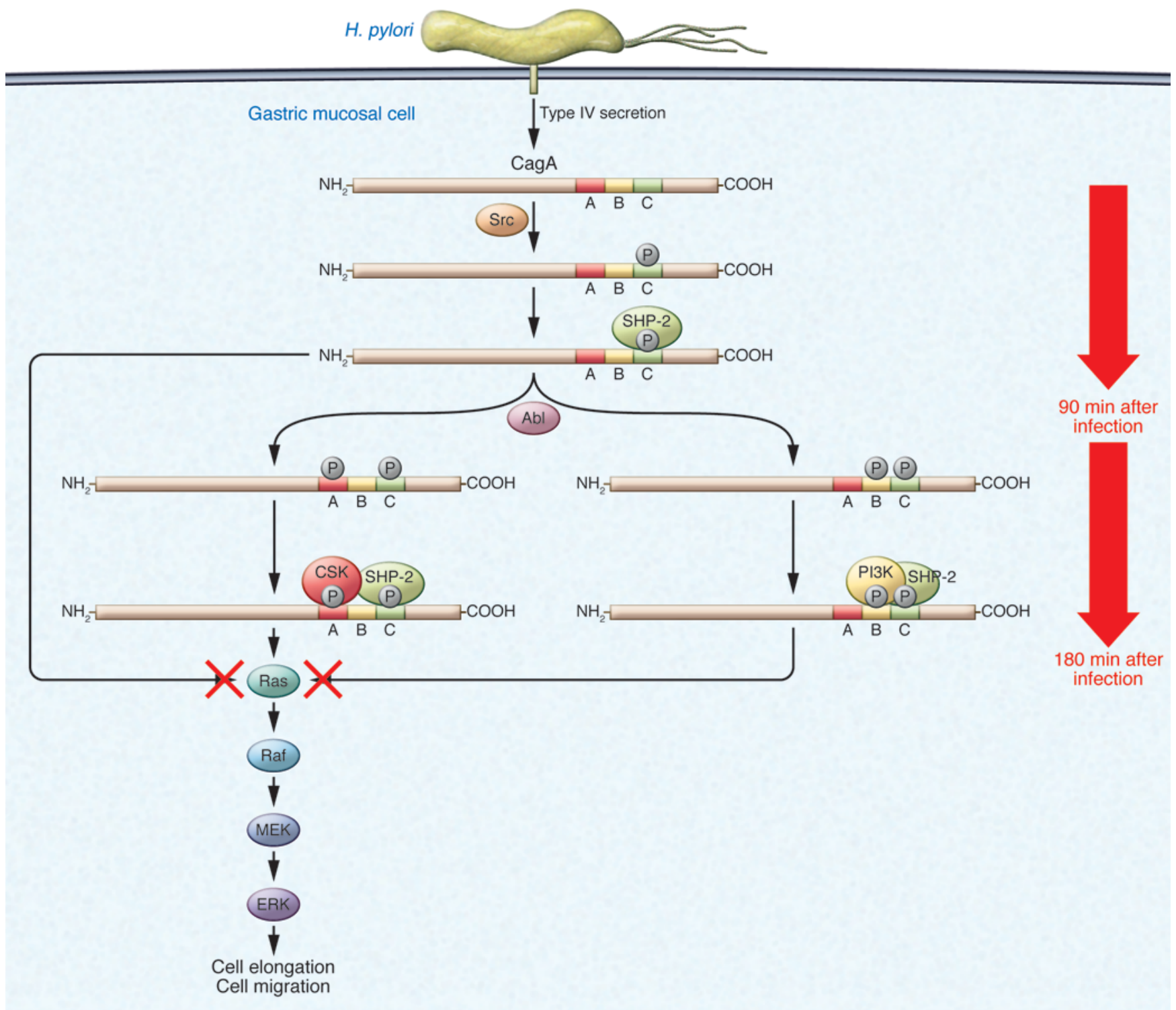

\section{Figure 2}

Simplified model showing the consecutive phosphorylation of EPIYA-C, -A, and -B motifs by c-Src and c-Abl kinases in the commonly occurring Western CagA EPIYA-ABC configuration, and subsequent downstream effects. Src family kinases become activated between 30 and 90 minutes after infection and exclusively phosphorylate the tyrosine residue of an EPIYA motif surrounded by $\mathrm{C}$ segment sequences. SHP-2 is known to bind to EPIYA-C ${ }^{P Y}$. EPIYA-C ${ }^{P Y}$ CagA becomes a substrate for Abl family kinases at 90-180 minutes after infection; c-Abl is in principle capable of phosphorylating tyrosine residues in EPIYA motifs located in either $\mathrm{A}, \mathrm{B}$, or $\mathrm{C}$ segments, but never phosphorylates more than 2 residues per molecule (preferentially $A$ and $C$ ). Triple-phosphorylated CagA is not detectable in infected cells. CagA ${ }^{P Y}$ interactors such as CSK and PI3K bind to EPIYA-APY and EPIYA-B ${ }^{P Y}$ motifs. Double-phosphorylated EPIYA-ACPY CagA triggers the activation of several downstream signaling pathways; the Ras-RafMEK pathway is shown representatively. The biological and cell morphological consequences of CagA phosphorylation are cell elongation and increased cell motility. Single-phosphorylated EPIYA-C ${ }^{P Y}$ CagA or double-phosphorylated EPIYA-BC ${ }^{P Y}$ CagA do not efficiently induce Ras-Raf-MEK signaling. In East Asian CagA variants, EPIYA-D functionally replaces EPIYA-C as the initial motif to be phosphorylated by c-Src (not shown).

tonella henselae, or effectors secreted via type III secretion systems, such as the Tir effector of enteropathogenic E. coli and the Tarp effector of Chlamydia trachomatis, remains to be determined in the future. As more than one phosphorylation site has been described for each of these virulence determinants (15), it seems likely that they are regulated by an analogous mechanism of tightly coordinated activation by host cell kinases.

Another point that remains a challenge for future experiments is the dissection of the relative contribution of each of the phosphorylation sites to clinical outcome. Aside from the most common EPIYA configura- tions (Figure 1), numerous less frequent $\mathrm{A}$, $\mathrm{B}$, and $\mathrm{C}$ combinations can be found in $H$. pylori clinical isolates. In light of the findings reported by Mueller et al. (18), it will be interesting to correlate these less common EPIYA configurations with the risk of developing gastric cancer or other severe outcomes of chronic $H$. pylori infection. With more and 
more studies now indicating that $H$. pylori infection can have clear beneficial effects on the development of asthma and other chronic allergic and autoimmune diseases $(20,21)$, the distinction between "good" and "bad" H. pylori strains is becoming increasingly important in a clinical setting. The decision of whether to treat a given individual to eradicate their $H$. pylori infection could potentially be guided by a detailed analysis of the CagA phosphorylation status, and, in my opinion, treatment should probably be reserved for symptomatic carriers of strains expressing virulent CagA variants.

\section{Acknowledgments}

A. Müller acknowledges financial support from the Swiss National Science Foundation, the Swiss Cancer League, the Zurich Cantonal Cancer League, and the Gebert Rüf Foundation.

Address correspondence to: Anne Müller, Institute of Molecular Cancer Research, University of Zürich, Winterthurerstr. 190, CH-8057 Zürich. Phone: 41.44.635.3474; Fax: 41.44.635.3484; E-mail: mueller@ imcr.uzh.ch.
55(2):74-108.

2. Parsonnet J, et al. Helicobacter pylori infection and the risk of gastric carcinoma. N Engl J Med. 1991; 325(16):1127-1131.

3. Huang JQ, Zheng GF, Sumanac K, Irvine EJ, Hunt RH. Meta-analysis of the relationship between cagA seropositivity and gastric cancer. Gastroenterology. 2003;125(6):1636-1644.

4. Parsonnet J, Friedman GD, Orentreich N, Vogelman $\mathrm{H}$. Risk for gastric cancer in people with CagA positive or CagA negative Helicobacter pylori infection. Gut. 1997;40(3):297-301.

5. Rieder G, Merchant JL, Haas R. Helicobacter pylori cag-type IV secretion system facilitates corpus colonization to induce precancerous conditions in Mongolian gerbils. Gastroenterology. 2005; 128(5):1229-1242.

6. Arnold IC, et al. Tolerance rather than immunity protects from Helicobacter pylori-induced gastric preneoplasia. Gastroenterology. 2011; 140(1):199-209.

7. Ohnishi N, et al. Transgenic expression of Helicobacter pylori CagA induces gastrointestinal and hematopoietic neoplasms in mouse. Proc Natl Acad SciU S A. 2008;105(3):1003-1008.

8. Censini S, et al. cag, a pathogenicity island of Helicobacter pylori, encodes type I-specific and diseaseassociated virulence factors. Proc Natl Acad SciUS A. 1996;93(25):14648-14653.

9. Odenbreit S, Puls J, Sedlmaier B, Gerland E, Fischer W, Haas R. Translocation of Helicobacter pylori CagA into gastric epithelial cells by type IV secretion. Science. 2000;287(5457):1497-1500.

10. Poppe M, Feller SM, Romer G, Wessler S. Phosphorylation of Helicobacter pylori CagA by c-Abl leads to cell motility. Oncogene. 2007;26(24):3462-3472.

11. Selbach M, Moese S, Hauck CR, Meyer TF, Backert S. Src is the kinase of the Helicobacter pylori CagA protein in vitro and in vivo. J Biol Chem. 2002;
277(9):6775-6778.

12. Higashi H, et al. Biological activity of the Helicobacter pylori virulence factor CagA is determined by variation in the tyrosine phosphorylation sites. Proc Natl Acad Sci U S A. 2002;99(22):14428-14433.

13. Higashi $\mathrm{H}$, et al. SHP-2 tyrosine phosphatase as an intracellular target of Helicobacter pylori CagA protein. Science. 2002;295(5555):683-686.

14. Tsutsumi R, Higashi H, Higuchi M, Okada M, Hatakeyama M. Attenuation of Helicobacter pylori CagA x SHP-2 signaling by interaction between CagA and C-terminal Src kinase. J Biol Chem. 2003; 278(6):3664-3670.

15. Selbach M, et al. Host cell interactome of tyrosinephosphorylated bacterial proteins. Cell Host Microbe. 2009;5(4):397-403.

16. Amieva MR, Vogelmann R, Covacci A, Tompkins LS, Nelson WJ, Falkow S. Disruption of the epithelial apical-junctional complex by Helicobacter pylori CagA. Science. 2003;300(5624):1430-1434.

17. Saadat I, et al. Helicobacter pylori CagA targets PAR1/ MARK kinase to disrupt epithelial cell polarity. Nature. 2007;447(7142):330-333.

18. Mueller D, et al. c-Src and c-Abl kinases control hierarchic phosphorylation and function of the CagA effector protein in Western and East Asian Helicobacter pylori strains. J Clin Invest. 2012; 122(4):1553-1566.

19. Patwardhan P, Miller WT. Processive phosphorylation: mechanism and biological importance. Cell Signal. 2007;19(11):2218-2226.

20. Blaser MJ, Chen Y, Reibman J. Does Helicobacter pylori protect against asthma and allergy? Gut. 2008;57(5):561-567.

21. Luther J, Dave M, Higgins PD, Kao JY. Association between Helicobacter pylori infection and inflammatory bowel disease: a meta-analysis and systematic review of the literature. Inflamm Bowel Dis. 2010;16(6):1077-1084.

\title{
Parkinson's disease: don't mess with calcium
}

\author{
Mark P. Mattson
}

\author{
Laboratory of Neurosciences, National Institute on Aging Intramural Research Program, Baltimore, Maryland, USA.
}

\begin{abstract}
The hallmark of the movement disorder Parkinson's disease (PD) is progressive degeneration of dopaminergic neurons. Mitochondrial dysfunction, impaired ubiquitin-mediated proteolysis of $\alpha$-synuclein, and ER stress are each implicated in the complex and poorly understood sequence of events leading to dopaminergic neuron demise. In this issue of the JCI, Selvaraj et al. report that in a mouse neurotoxin-based model of $\mathrm{PD}$, reduced $\mathrm{Ca}^{2+}$ influx through transient receptor potential C1 (TRPC1) channels in the plasma membrane of dopaminergic neurons triggers a cell death-inducing ER stress response. These new findings suggest that TRPC1 channels normally function in $\mathrm{Ca}^{2+}$-mediated signaling pathways that couple adaptive/neurotrophic responses to metabolic and oxidative stress and suggest that disruption of these pathways may contribute to PD.
\end{abstract}

\section{Introduction}

Approximately one million Americans have Parkinson's disease (PD), a fatal neurode-

Conflict of interest: The author has declared that no conflict of interest exists.

Citation for this article: J Clin Invest. 2012; 122(4):1195-1198. doi:10.1172/JCI62835. generative disorder that involves progressive dysfunction and death of neurons in the brain stem, midbrain, and cerebral cortex. The tremors and rigidity that typify PD result from degeneration of neurons in the substantia nigra that normally produce and release the neurotransmitter dopamine. Despite this long-standing knowledge, the sequence of events leading to dopaminergic neuron demise is complex and poorly understood. PD most commonly manifests late in life and is sporadic, suggesting an important etiologic role for environmental factors. There are, however, rare cases of PD caused by mutations in the genes encoding $\alpha$-synuclein, leucine-rich repeat kinase 2 (LRRK2), Parkin, PTEN-induced putative kinase 1 (PINK1), or DJ-1 (1). In most cases of PD, $\alpha$-synuclein aggregates and accumulates inside vulnerable neurons, and this is thought to be a crucial component of disease pathogenesis.

A link between mitochondrial dysfunction and the demise of dopaminergic neurons in PD has been recognized for several decades. Early insight came from an incident in California in which several individuals with drug addiction presented with a PD-like syndrome of rapid onset as a result of unwitting ingestion of the 